\title{
The Problem of Renal Function Monitoring in Patients Treated With the Novel Antiretroviral Drugs
}

\author{
Paolo Maggi, ${ }^{1}$ Vincenzo Montinaro, ${ }^{2}$ Stefano Rusconi, ${ }^{3}$ Antonio Di Biagio, ${ }^{4}$ Rita Bellagamba, ${ }^{5}$ \\ Paolo Bonfanti, ${ }^{6}$ Leonardo Calza, ${ }^{7}$ Paola Corsi, ${ }^{8}$ Francesco Montella, ${ }^{9}$ and Cristina Mussini ${ }^{10}$
}

${ }^{1}$ Clinic of Infectious Diseases, University Hospital "Policlinico", Bari, Italy; ${ }^{2}$ Division of Nephrology, University Hospital "Policlinico", Bari, Italy; 'Infectious Disease and Immunopathology Unit, Department of Clinical Sciences "Luigi Sacco", University of Milano, Italy; ${ }^{4}$ Clinic of Infectious Disease, University Hospital "San Martino", Genova, Italy; Infectious and Tropical Diseases Unit, National Institute for Infectious Disease "L. Spallanzani”, Roma, Italy; ${ }^{6}$ Infectious Diseases Unit, Hospital "A.Manzoni", Lecco, Italy; ${ }^{7}$ Clinic of Infectious Disease, University Hospital "S.Orsola-Malpighi”, Bologna, Italy; 'Infectious Disease Unit, University Hospital "Careggi", Firenze, Italy; IInternal Medicine and Clinical Immunology Unit, Hospital "San Giovanni", Roma, Italy; ${ }^{10} \mathrm{Clinic}$ of Infectious Disease, University Hospital, Modena, Italy

Chronic kidney disease (CKD) is currently considered a major comorbidity in patients affected by HIV infection. In addition, new generation antiretroviral drugs that interact with creatinine transporters were recently introduced. Rilpivirine, dolutegravir, and cobicistat, with different mechanisms, inhibit the amount of tubular secretion of creatinine causing a slight increase in serum creatinine levels and consensual eGFR $_{\text {creat }}$ reduction. This will require an unprecedented attention to renal issues, because the new drugs can also be associated to old antiretroviral drugs that may exert renal toxic effects. Owing to the interference of these drugs with creatinine secretion, an alternative way of estimating GFR would be desirable. At the moment, methods of direct GFR measurement have a high impact on the patient, are not readily available, or are not reliable in HIV patients. Consequently, use of classic formulas to estimate GFR is still recommended, considering the apparent reduction of eGFR $_{\text {creat }}$ due to these drugs. Tubular function needs to be carefully monitored with simple tests such as proteinuria, phosphatemia, urinary excretion of phosphate, normoglycemic glycosuria, and excretion of uric acid. More specific and sensitive markers of tubular damage are still not readily available in all clinical labs. HIV patients treated by the novel drugs need to be monitored on a monthly basis for the first 3 months. Subsequent monitoring should be performed on a quarterly basis or guided by comorbidities. Key words: cobicistat, dolutegravir, HIV, kidney, rilpivirine

\section{CHRONIC KIDNEY DISEASE AND HIV INFECTION}

Chronic kidney disease (CKD) is currently considered a major comorbidity in patients who are affected by HIV infection. ${ }^{1,2}$ The increase in life expectancy that followed the introduction of combination antiretroviral therapy (cART) induced the emergence of common chronic conditions, such as diabetes and hypertension, that act as major pathogenic determinants for the development of CKD. ${ }^{2,3}$ Moreover, chronic inflammation caused by the infection and probably direct renal damage mediated by viral products on kidney biology are contributory factors for the onset of CKD in these subjects. Finally, the potential toxic effect of some antiretroviral agents, such as indinavir or tenofovir (TDF), is well known with a body of evidence. ${ }^{4-6}$
Although these drugs may rarely induce clinically evident nephrotoxic renal damage, they more often represent a cofactor in the determinism of CKD.

\section{NOVEL ANTIRETROVIRAL DRUGS}

In addition to this complex scenario, new antiretroviral drugs that interact with creatinine transporters have recently become available. These drugs present a good safety and tolerability profile,

Address for correspondence: Paolo Maggi, Department of Infectious Disease, University of Bari-Policlinico, Piazza Giulio Cesare, 11, Bari 70214 Italy; e-mail: p_maggi@yahoo.com

HIV Clin Trials 2014;15(3):87-91

(C) 2014 Thomas Land Publishers, Inc.

www.hivclinicaltrials.com

doi: 10.1310/hct1503-87 
which gives them better security and manageability compared to older drugs. However, some apparent concerns may arise from their reported effects on renal function.

The first of these compounds is rilpivirine, a nonnucleoside reverse transcriptase inhibitor (NNRTI). Rilpivirine has a high protein-binding capacity and is excreted only minimally by the kidney.

In 2 large trials, rilpivirine determined a consistent increase of serum creatinine ( $\mathrm{SCr}$ ) (an average of approximately $0.1 \mathrm{mg} / \mathrm{dL}$ ) in patients with normal renal function. ${ }^{7,8}$ This increase was stable over time and was not associated with other laboratory parameters of kidney damage; in turn, it affected GFR estimation based on creatinine (eGFR creat $_{\text {e }}$ ).

Rilpivirine has been reported to act as an inhibitor of the renal organic cationic transporter (OCT2) at the basolateral side of proximal renal tubular cells (Figure 1) ${ }^{9}$ in a way that mimics the effects of cimetidine, quinidine, or trimethoprim; all these drugs decrease the secretion of creatinine by the proximal renal tubule. ${ }^{10}$ GFR estimated by formulas based on serum cystatin c $\left(\mathrm{eGFR}_{\text {cys }}\right.$ ) does not show the above-mentioned reduction under rilpivirine therapy. However, caution in using these formulas is suggested, because of possible variations in cystatin c generation according to the virological status of the patient. ${ }^{11}$

Dolutegravir is a novel integrase inhibitor that is metabolized by hepatic UGT1A1 with a minor role of CYP3A. Renal elimination of unchanged dolutegravir is less than $1 \% .{ }^{12}$ In a comparison study of dolutegravir versus raltegravir (SPRING), a small increase in creatinine was observed in the dolutegravir arm. ${ }^{13}$ This slight, nonprogressive, initial increase in $\mathrm{SCr}$ by dolutegravir is in the range of 0.1 to $0.15 \mathrm{mg} / \mathrm{dL}$ and has been demonstrated not to affect actual glomerular filtration rate (a-GFR), measured by the iohexol clearance. ${ }^{14,15}$

In this case, in vitro and clinical data are consistent with inhibition of the OCT2. ${ }^{12}$ This transporter is positioned at the basolateral side of the proximal renal tubular cells and is responsible for the uptake of creatinine by the proximal renal tubular cells from

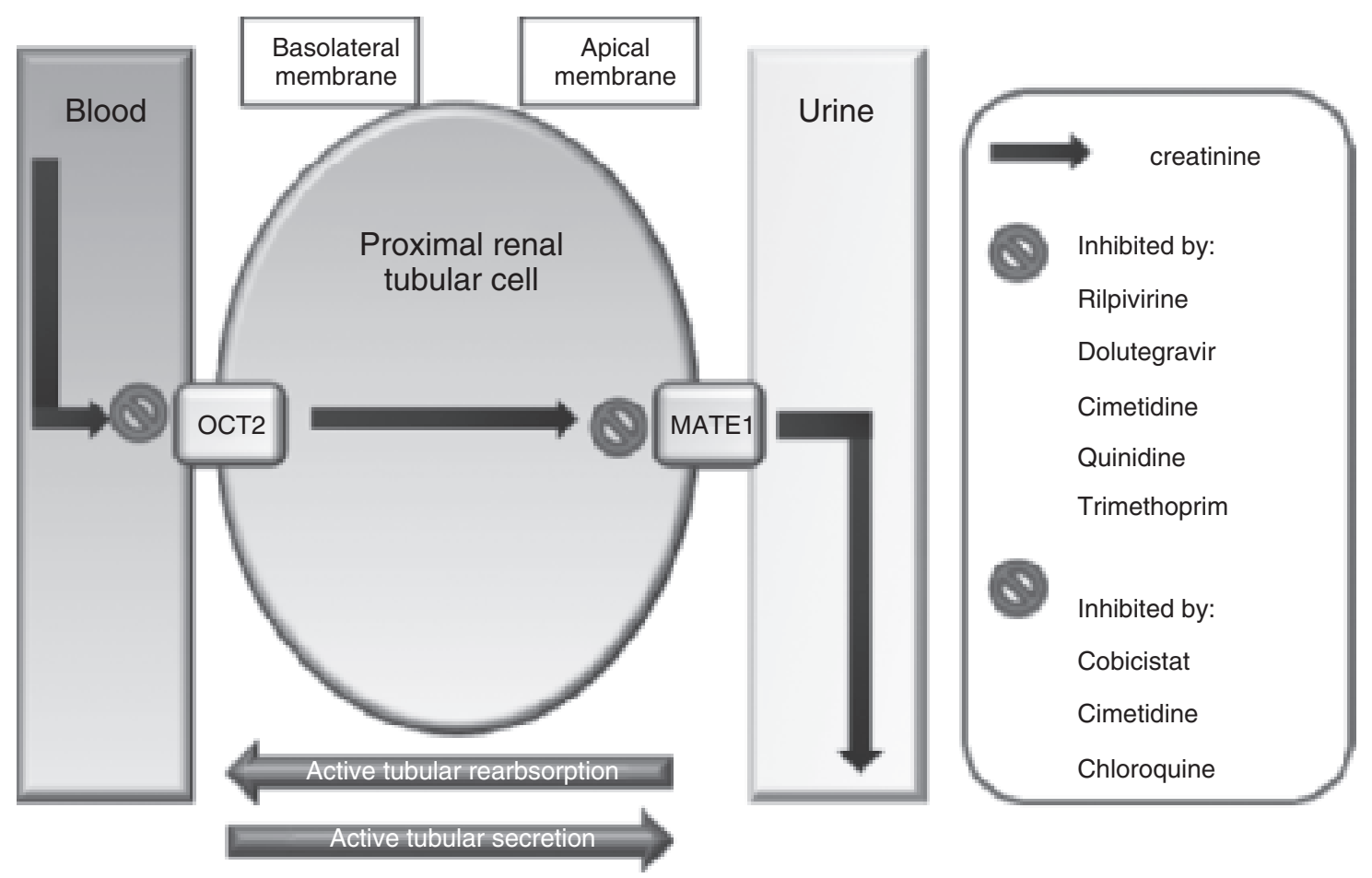

Figure 1. Renal tubular transporters. Urinary creatinine is secreted by tubule at approximately $10 \%$ of total amount. Creatinine is an endogenous substrate of OCT2 (uptake in tubule cells). ${ }^{10,32}$ Creatinine efflux in urine seems mediated by MATE1 and MATE2-K. 
blood. Competitive pharmacological inhibition of OCT2 halts tubular secretion of creatinine (Figure 1).

Cobicistat is a new pharmacokinetic enhancer, metabolized by CYP3A, that does not have any antiretroviral property per se. ${ }^{16}$ Cobicistat provokes an increase in SCr and a decrease in eGFR based on creatinine measurement. Previous research has demonstrated that cobicistat is a potent inhibitor of MATE1 (Human Multidrug and Toxic Extrusion 1 ), which is present at the apical side of proximal renal tubular cells and is responsible for the efflux of creatinine from the tubular cells ${ }^{17}$ (Figure 1). Therefore, cobicistat inhibits the amount of tubular secretion of creatinine and causes a slight $(<10 \%)$ increase in serum creatinine levels and consensual eGFR $_{\text {creat }}$ reduction, without other evidence of kidney damage. This laboratory changes are promptly reversible upon drug withdrawal.

\section{PROBLEM OF RENAL FUNCTION MONITORING}

Based on all the considerations about the new antiretroviral drugs, the introduction of these various therapeutic agents requires that careful attention is paid to renal issues. Also, the new drugs can be associated to old antiretroviral drugs that, under certain conditions, may exert renal toxic effects. Therefore, patients who will be exposed to rilpivirine, cobicistat, and dolutegravir must be monitored with simple and reliable parameters of renal dysfunction, including indicators of GFR and tubular damage.

Owing to the interference of these new drugs with tubular creatinine secretion, it would be desirable to have an alternative way of estimating GFR. However, at the moment, the issue cannot be easily resolved. On the one hand, methods of direct GFR measurement, such as inuline, iohexol, or iothalamate plasma or renal clearances, would help overcome this drawback. Nevertheless, all these methods have a high impact on the patient, require repeated blood sampling, and are not readily available in common clinical labs because they require complex analytical procedures. On the other hand, formulas that estimate GFR and are based on cystatin $\mathrm{C}$, even though they have good performance in the general population, ${ }^{18}$ are not useful in HIV patients, because production of cystatin $C$ is not constant during the different phases of the infection. Patients with detectable viremia and low CD4 count tend to have a higher production of this molecule, with concomitant higher plasma levels..$^{19-22}$
After initiating the cART, with reduction and possibly disappearance of HIV RNA from circulation and an increase in CD4 cells, the cystatin C production rate declines. As a result, patients starting their first cART treatment show an increase of eGFR of 20 to $25 \mathrm{~mL} / \mathrm{min}$ when they change from the initial viremic and systemic inflamed state to a chronic nonviremic condition; this increment is mostly apparent and is at least in part due to the reduced serum levels of cystatin C. ${ }^{21}$

\section{MANAGEMENT OF HIV PATIENTS TREATED WITH THE NOVEL DRUGS}

GFR can be estimated by applying validated formulas such as the CKD-EPI based on creatinine to monitor renal function in patients treated with one of the above-mentioned drugs. ${ }^{23}$ Antiretroviral regimens that include rilpivirine, dolutegravir, or cobicistat will produce an apparent reduction of eGFR that, in normal conditions, should not exceed $25 \%$ compared with the basal level. A greater reduction

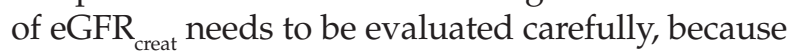
it may represent a sign of true renal function impairment. Patients who have a basal GFR lower than 60 $\mathrm{mL} / \mathrm{min} / 1.73 \mathrm{~m}^{2}$ may experience an even higher reduction of eGFR, owing to the proportionally greater tubular secretion that accounts for creatinine excretion in conditions of moderate renal impairment. In these conditions, tubular secretion may be higher than $35 \%$ of total excretion. ${ }^{24}$

In addition to the evaluation of glomerular filtration, the tubular function needs to be carefully monitored. Simple tests may help manage this issue. Proteinuria should always be looked for in patients who are exposed to potential nephrotoxic drugs. Dipstick proteinuria has a high negative predictive value, but a low positive predictive value. ${ }^{25}$ It can only be a screening test. Any patient with an altered dipstick proteinuria needs a more accurate evaluation. We recommend performing quantitative proteinuria estimation as total protein and albumin over creatinine ratio (PCR and ACR, respectively). These measurements can be done on morning spot urine samples, which avoids the burden of timely collected urine. ACR greater than $30 \mathrm{mg} / \mathrm{g}$ indicates moderately increased albuminuria; if it is greater than $300 \mathrm{mg} / \mathrm{g}$, it is a sign of severe albuminuria or frank proteinuria. ${ }^{26}$ PCR higher than $150 \mathrm{mg} / \mathrm{g}$ is also an altered value. The ratio between ACR and PCR may help discriminate between glomerular and tubular proteinuria. A ratio greater than 0.4 
indicates glomerular proteinuria, while a ratio lower than 0.4 is a marker of tubular proteinuria. ${ }^{27}$

Tubular proteinuria can also be detected by specific markers such as urinary excretion of retinol binding protein, $\alpha-1$-microglobulin, $\beta-2-$ microblobulin, and NGAL (neutrophil gelatinaseassociated lipocalin). ${ }^{28}$ These are sensitive markers of tubular damage, yet are not readily available in all clinical labs.

Tubular dysfunction can also be assessed as an increased urinary excretion of phosphate, better estimated as the $\mathrm{TmPO}_{4} /$ GFR ratio and as hypophosphatemia $(<2.5 \mathrm{mg} / \mathrm{dL}) .{ }^{29} \mathrm{An}$ increased fractional excretion of uric acid can also be easily assessed and is a marker of early tubular damage. Finally, normoglycemic glycosuria appears as an advanced sign of tubular dysfunction. ${ }^{30}$

We suggest that HIV patients who are treated by potential nephrotoxins, especially in association with the novel drugs that affect creatinine excretion, need to be monitored on a monthly basis for the first 3 months. Patients who experience a decrease of eGFR creat greater than $25 \%$ or show the de novo occurrence of signs of tubular dysfunction need a nephrological consult. Generally, new abnormalities attenuate after week 16 of cART. ${ }^{31}$ Consequently, for abnormalities that are not present by week 16, subsequent monitoring should be performed on a quarterly basis or guided by comorbidities.

In conclusion, the introduction of new antiretroviral agents that potentially affect renal function and their foreseeable wide therapeutic application in the near future is raising some concerns. Clinicians should carefully monitor renal function to identify possible alterations suggestive of a true renal functional impairment. Patients should also be monitored for the presence of other risk factors for kidney disease, such as growing age of HIV patients and putative nephrotoxicity of other drugs in the ARV regimens. Results from clinical trials conducted with these novel agents are in some way reassuring, but physicians in routine clinical practice will need evaluation strategies for the long-term surveillance of eventual toxicities.

\section{ACKNOWLEDGMENTS}

We are grateful to Ms. Paulene Butts for her assistance in the preparation of the manuscript.

\section{REFERENCES}

1. Maggi P, Bartolozzi D, Bonfanti P, et al. Renal complications in HIV disease: Between present and future. AIDS Rev. 2012;14:37-53.

2. Ryom L, Mocroft A, Kirk O, et al. Predictors of advanced chronic kidney disease and end-stage renal disease in HIV-positive persons. AIDS. 2014;28(2):187-199.

3. Margolick JB, Jacobson LP, Schwartz GJ, et al. Factors affecting glomerular filtration rate, as measured by iohexol disappearance, in men with or at risk for HIV infection. PLoS One. 2014;9(2):e86311.

4. Danjuma MI, Mohamad-Fadzillah NH, Khoo S. An investigation of the pattern of kidney injury in HIV-positive persons exposed to tenofovir disoproxil fumarate: An examination of a large population database (MHRA database). Int J STD AIDS. 2014;25(4):273-279.

5. Maggi P, Montinaro V, Bellacosa C, et al . Early markers of tubular dysfunction in antiretroviral-experienced HIVinfected patients treated with tenofovir versus abacavir. AIDS Patient Care STDS. 2012;26(1):5-11.

6. Flandre P, Pugliese P, Cuzin L, et al; New AIDS Data Group. Risk factors of chronic kidney disease in HIV-infected patients. Clin J Am Soc Nephrol. 2011;6(7):1700-1707.

7. Molina JM, Cahn P, Grinsztejn B, et al; ECHO Study Group. Rilpivirine versus efavirenz with tenofovir and emtricitabine in treatment-naive adults infected with HIV-1 (ECHO): A phase 3 randomised double-blind active-controlled trial. Lancet. 2011;378(9787):238-246.

8. Cohen CJ, Andrade-Villanueva J, Clotet B, et al; THRIVE Study Group. Rilpivirine versus efavirenz with two background nucleoside or nucleotide reverse transcriptase inhibitors in treatment-naive adults infected with HIV-1 (THRIVE): A phase 3, randomised, non-inferiority trial. Lancet. 2011;378(9787):229-237.

9. Cohen CJ, Molina JM, Cassetti I, et al; ECHO, THRIVE Study Groups. Week 96 efficacy and safety of rilpivirine in treatment-naive, HIV-1 patients in two Phase III randomized trials. AIDS. 2013;27(6):939-950.

10. Urakami Y, Kimura N, Okuda M, Inui K. Creatinine transport by basolateral organic cation transporter hOCT2 in the human kidney. Pharm Res. 2004;21(6):976-981.

11. Gagneux-Brunon A, Mariat C, Delanaye P. Cystatin $C$ in HIV-infected patients: Promising but not yet ready for prime time. Nephrol Dial Transplant. 2012;27(4):1305-1313.

12. Reese MJ, Savina PM, Generaux GT, et al. In vitro investigations into the roles ofdrug transporters and metabolizing enzymes in the disposition and druginteractions of dolutegravir, a HIV integrase inhibitor. Drug Metab Dispos. 2013;41(2):353-361.

13. Raffi F, Rachlis A, Stellbrink HJ, et al. Once-daily dolutegravir versus raltegravir in antiretroviral-naive adults with HIV-1 infection: 48 week results from the randomised, double-blind, non-inferiority SPRING-2 study. Lancet. 2013;381:735-774.

14. Koteff J, Borland J, Chen S et al. A phase 1 study to evaluate the effect of dolutegravir on renal function via measurement of iohexol and para-aminohippurate clearance in healthy subjects. Br J Clin Pharmacol. 2012;75:990-996.

15. Stellbrink HJ, Reynes J, Lazzarin A, et al. Dolutegravir (DTG; S/GSK1349572) in combination therapy exhibits rapid and 
sustained antiviral response in antiretroviral-naïve adults: 96-week results from SPRING-1 (ING112276). Presented at: 19th CROI; March 5-8, 2012. Abstract K-102.

16. Mathias AA, German P, Murray BP, et al. Pharmacokinetics and pharmacodynamics of GS-9350: A novel pharmacokinetic enhancer without anti-HIV activity. Clin Pharmacol Ther. 2010;87(3):322-329.

17. Meyer zu Schwabedissen HE, Verstuyft C, Kroemer HK, et al. Human multidrug and toxin extrusion 1 (MATE1/SLC47A1) transporter: Functional characterization, interaction with OCT2 (SLC22A2), and single nucleotide polymorphisms. Am J Physiol Renal Physiol. 2010;298(4):F997-F1005.

18. Stevens LA, Coresh J, Schmid CH, et al. Estimating GFR using serum cystatin $\mathrm{C}$ alone and in combination with serum creatinine: A pooled analysis of 3,418 individuals with CKD. Am J Kidney Dis. 2008;51:395-406.

19. Jaroszewicz J, Wiercinska-Drapalo A, Lapinski TW, et al. Does HAART improve renal function ? An association between serum cystatin C concentration, HIV viral load and HAART duration. Antivir Ther. 2006;11:641-645.

20. Longenecker CT, Scherzer R, Bacchetti $P$, et al. HIV viremia and changes in kidney function. AIDS. 2009;23:10891096.

21. Mauss S, Berger F, Kuschak D, et al. Cystatin C as a marker of renal function is affected by HIV replication leading to an underestimation of kidney function in HIV patients. Antivir Ther. 2008;13:1091-1095.

22. Odden MC, Scherzer R, Bacchetti P, et al. Cystatin C level as a marker of kidney function in human immunodeficiency virus infection: The FRAM study. Arch Intern Med. 2007; 167:2213-2219

23. Inker LA, Wyatt C, Creamer R, et al. Performance of creatinine and cystatin C GFR estimating equations in an HIV- positive population on antiretrovirals. J Acquir Immune Defic Syndr. 2012;61(3):302-309.

24. Delanaye P, Mariat C, Cavalier E, Maillard N, Krzesinski JM, White CA. Trimethoprim, creatinine and creatinine-based equations. Nephron Clin Pract. 2011;119(3):187-193.

25. Atkins RC, Briganti EM, Zimmet PZ, Chadban SJ. Association between albuminuria and proteinuria in the general population: The AusDiab Study. Nephrol Dial Transplant. 2003;18(10):2170-2174.

26. KDIGO clinical practice guideline for the evaluation and management of chronic kidney disease. Kidney Int. 2013;3(Suppl):1-150.

27. Samarawickrama A, Cai M, Smith ER, et al. Simultaneous measurement of urinary albumin and total protein may facilitate decision-making in HIV-infected patients with proteinuria. HIV Med. 2012;13:526-532.

28. del Palacio M, Romero S, Casado JL. The use of biomarkers for assessing HAART-associated renal toxicity in HIV- infected patients. Curr HIV Res. 2012;10(6):521-531.

29. Imel EA, Econs MJ. Approach to the hypophosphatemic patient. J Clin Endocrinol Metab. 2012;97(3):696-706.

30. Herlitz LC, Mohan S, Stokes MB, et al. Tenofovir nephrotoxicity: Acute tubular necrosis with distinctive clinical, pathological, and mitochondrial abnormalities. Kidney Int. 2010;78(11):1171-7.

31. Taiwo B, Yanik EL, Napravnik S, et al. Evidence for risk stratification when monitoring for toxicities following initiation of combination antiretroviral therapy. AIDS. 2013;27(10):1593-1602.

32. Imamura $\mathrm{Y}$, Murayama N, Okudaira $\mathrm{N}$ et al. Prediction of fluoroquinolone-induced elevation in serum creatinine levels: A case of drug-endogenous substance interaction involving the inhibition of renal secretion. Clin Pharmacol Ther. 2011;89(1):81-88. 